\title{
A novel surgical approach for the treatment of tumors in the lesser trochanter
}

\author{
YIPIN YU ${ }^{1 *}$, XUEBIN SUN ${ }^{2 *}$, XINHUA SONG ${ }^{2}$, ZHENG TIAN $^{2}$ and YIJUN ZHOU ${ }^{2}$ \\ ${ }^{1}$ Department of Spinal Surgery, The Second Xiangya Hospital, Central South University, Changsha, Hunan 410011; \\ ${ }^{2}$ Department of Orthopedics, The First Affiliated Hospital of Xinjiang Medical University, \\ Ürümqi, Xinjiang 830054, P.R. China
}

Received June 1, 2014; Accepted February 5, 2015

DOI: $10.3892 / \mathrm{etm} .2015 .2453$

\begin{abstract}
The proximal femur is a common location for the development of primary benign bone tumors. However, there is currently no surgical technique designed specifically for treating tumors located in the lesser trochanter. In the present study, a novel procedure was developed for directly exposing the lesser trochanter for surgical intervention. This technique may be particularly suited to treating tumors that extend toward the lateral and anterior forward of the lesser trochanter. The new approach involved passing through the femoral triangle, separating the femoral nerve and femoral vessels (artery and vein) and resecting the tumor between the iliopsoas and pectineal muscles. The procedure was performed on six patients with various types of tumor, including one case with osteoid osteoma, one case with non-osteogenic fibroma, one case with osteoma, one case with liposarcoma and two cases of osteochondroma. The preliminary results indicated that the surgical durations were short (60-100 $\mathrm{min})$, blood loss was minimal $(30-200 \mathrm{ml})$ and that pain relief was achieved following surgery. Only one patient continued to experience mild pain, scoring $18 \mathrm{~mm}$ on a visual analog scale. The other patients were fully relieved of pain. Sensory dysfunction was experienced by one patient following surgery, with persistent numbness and paresthesias in the distribution of the femoral nerve. No cases of deep vein thrombosis, femur head necrosis, hip joint degeneration disease or local recurrence were identified in any patients during the follow-up period. In order to clarify the virtual tissue, such vessels, nerves and the available space in our approach area, we collected 20 cadaveric specimens and performed anatomical examinations in and around the formal triangle. The spaces between the femoral artery
\end{abstract}

Correspondence to: Dr Zheng Tian, Department of Orthopedics, The First Affiliated Hospital of Xinjiang Medical University, 137 Liyushan South Road, Ürümqi, Xinjiang 830054, P.R. China E-mail: tianzhengcn@126.com

${ }^{*}$ Contributed equally

Key words: bone neoplasm, surgical approach and femoral nerve were measured and analyzed, with the results demonstrating that a definite space existed. Therefore, the novel approach presented in the study may be useful in the resection of benign tumors and the preoperative palliative resection of malignant tumors. The technique may be particularly suited to tumors extending toward the lateral and anterior of the lesser trochanter.

\section{Introduction}

The proximal femur is one of the most common locations for primary benign bone tumor formation. The majority of benign tumors, including osteochondromas and fibrous dysplasia, remain asymptotic and do not require surgical intervention. However, there are a number of reasons why surgery may be performed, including pain, inhibited growth, decreased range of motion, cosmesis, peripheral nerve compression, vessel compression and to reduce the risk of a malignant transformation, with pain being the most common $(1,2)$. The recommended surgical intervention in these instances is the resection of the tumor (3). Numerous studies have focused on exposing the hip joint capsule or the femur neck, as tumors located in these areas more commonly lead to clinical symptoms and severe complications $(4,5)$. By contrast, tumors located in the lesser trochanter, particularly those outside of the hip joint capsule, may manifest with clinical symptoms gradually with a long asymptomatic period, and are seldom encountered in routine clinical practice. The conventional surgical intervention in these cases is to approach the lesser trochanter indirectly via the hip joint, which has various advantages and disadvantages. The conventional lateral approach is safe, but requires extreme rotation of the limb in order to expose enough of the region to perform surgery. By contrast, the medial approach, which provides the shortest approach to the hip, presents a challenge as it involve navigating complicated anatomical structure, including the highly variable obturator nerve, in order to expose the lesser trochanter (6).

Following detailed anatomical dissections, a novel and direct approach for the treatment of tumors in the lesser trochanter was developed. This technique involves passing through the femoral triangle and across the gap between the femoral artery and femoral nerve. The aim of the present study was to demonstrate the use of this novel approach, which may 

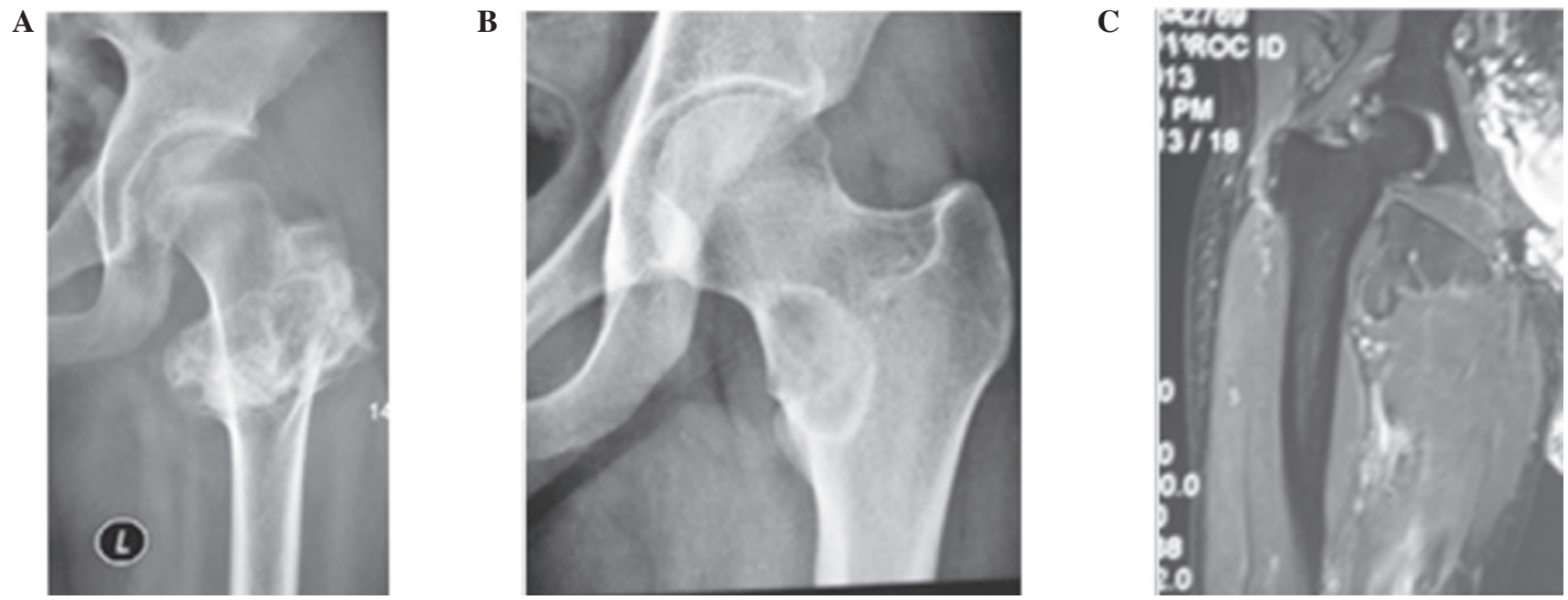

Figure 1. (A) Osteochondroma, (B) non-osteogenic fibroma and (C) liposarcoma located around the lesser trochanter.

be specifically suited to tumors projecting toward the lateral and anterior of the lesser trochanter. In addition, follow-up examination was performed to evaluate the outcome of the surgery in the patients.

\section{Materials and methods}

Clinical research. A total of six patients with tumors located in the lesser trochanter (Fig. 1) were treated at the First Affiliated Hospital of Xinjiang Medical University (Ürümqi, China) between March 2007 and March 2013. There were two male and four female patients, with a median age of 24 years (age range, 13-85 years). Prior to surgery, all the patients experienced mild to moderate pain, however, their ranges of motion around the hip joint were not affected. In four cases, the patient had experienced symptoms for over two years. A biopsy was performed on the patients $(5 / 6)$ prior to open surgery. There were two cases of osteochondroma and one case each of osteoid osteoma, non-osteogenic fibroma, osteoma and liposarcoma. The patients with an osteoma and liposarcoma had recurring tumors, and had previously received treatment in a different hospital. This study was conducted in accordance with the Declaration of Helsinki, and with approval from the Ethics Committee of Xinjiang Medical University. Written informed consent was obtained from all the participants prior to surgery.

A visual analog scale was used to evaluate the degree of pain experienced by the patients prior to surgery and at four weeks following surgery. All six patients underwent follow-up observation, which varied between 6 and 34 months after surgery; the median follow-up time was 25 months. Images were collected at 1, 3, 6, 12 and 24 months after surgery in order to evaluate local recurrence, femur head necrosis and the occurrence of degeneration disease. Radiographic images were obtained using a DirectView DR 3000 (Kodak, Rochester, NY, USA). All patients were subjected to hip magnetic resonance imaging (MRI) using a MAGNETOM Avanto 1.5T MRI scanner (Siemens AG, Munich, Germany). MRI examination was conducted at approximately the time of the patients' hospitalization. A conventional MRI sequence protocol was applied in all of the participants and fat saturation was applied in the patient with liposarcoma. Scanning parameters were as

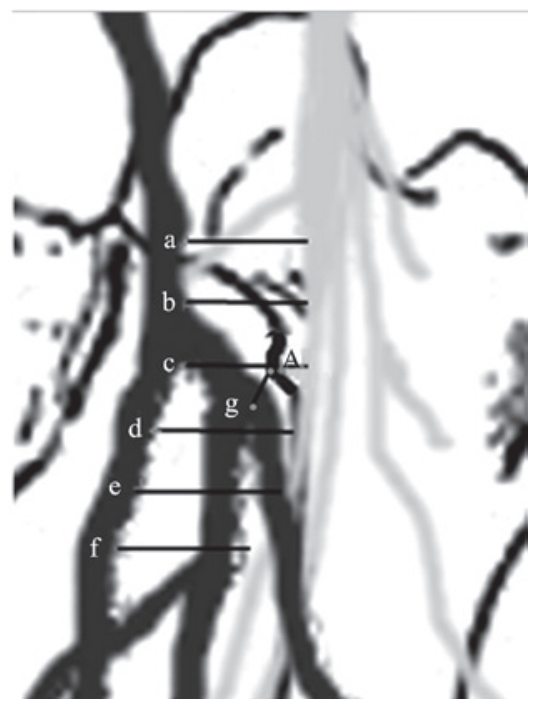

Figure 2. (A) Highest point of the lesser trochanter; (a) proximal distance from the lesser trochanter $(4 \mathrm{~cm})$; (b) proximal distance from the lesser trochanter $(2 \mathrm{~cm})$; (c) proximal distance from the lesser trochanter $(0 \mathrm{~cm})$; (d) distal distance from the lesser trochanter $(2 \mathrm{~cm})$; (e) distal distance from the lesser trochanter $(4 \mathrm{~cm})$; (f) distal distance from the lesser trochanter $(6 \mathrm{~cm}) ;(\mathrm{g})$ Distance from the highest point of lesser trochanter to the beginning of the lateral circumflex femoral artery.

follows: T1-weighted images had a repetition time of $600 \mathrm{msec}$ and an echo time of $10 \mathrm{msec}$; T2-weighted image repetition time was $4,000 \mathrm{msec}$ and the echo time was $110 \mathrm{msec}$. These materials were analyzed by two bone tumor specialists and one radiologist.

Cadaveric dissections. A total of 20 adult lower limb specimens without organic disease, including 5 unilateral and 4 bilateral male specimens, and 3 unilateral and 2 bilateral female specimens, were provided by the Department of Human Anatomy of Xinjiang Medical University. The virtual area of our approach was the formal triangle, where the formal artery and vein are located. The formal artery and vein are wrapped and limited by the surrounding tunica vaginalis, thus we focused on the gap between the lateral and medial margins of the femoral nerve. This gap is located by the innermost branch 

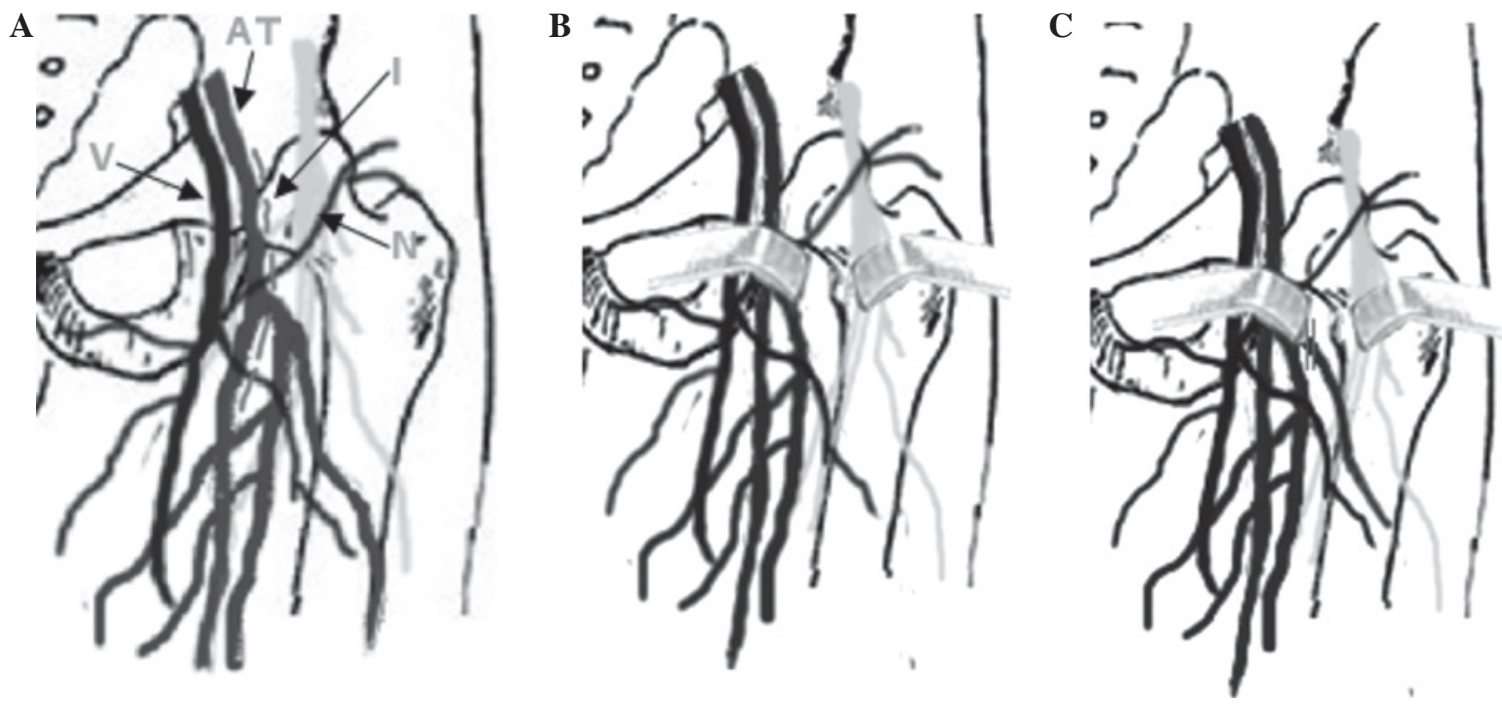

Figure 3. (A) The incision was centered over the tumor by the outside of the arteria femoralis. Arrow labels: AT, femoral artery; V, femoral vein; N, femoral nerve, I, incision. (B) The vessels including arteries and veins were trapped and protected towards medial, while nerves pulled toward lateral. (C) The lateral femoral circumflex artery and its branches do not always prevent further separation, but should be removed when this is the case.

among the branches of femoral nerve. In order to determine the space available for the surgical procedure, the distance of the gap described was measured. The proximal $(4,2$ and $0 \mathrm{~cm})$ and distal $(2,4$ and $6 \mathrm{~cm})$ distances were measured from the highest point of the lesser trochanter. In addition, the distance between the highest point of the lesser trochanter to the start of the lateral circumflex femoral artery was measured (Fig. 2). Measurements were calculated with vernier calipers (accuracy of $0.02 \mathrm{~mm}$ ) and were recorded twice.

Surgical technique. Preoperative assessment included identifying the position and extent of the tumors using imaging techniques. The first stage of the surgical procedure was to place the patient in the supine position, with the hip joint of the affected side facing outward in order to limit rotation. The pulse of the arteria femoralis was clearly identifiable in the femoral triangle region, near the tumor site. The incision was centered over the tumor, by the outside of the arteria femoralis, and the incision length could be adjusted (Fig. 3A). The superficial fascia incision was performed in the same manner as the standard surgical procedure. The deep fascia incision was made with caution, as a number of vital blood vessels and nerves associated with the femur ran through the area. The incision separated the tissue, with the blood vessels, nerves and surrounding adipose tissue separated to either side of the incision. Adipose tissue between the vessels and nerves was appropriately separated. Vessels, including arteries and veins, were trapped and protected away from the incision area towards the medial, while nerves were pulled laterally (Fig. 3B). Further separation was performed through the adipose tissue. Small branches of the blood vessels and the femoral nerves were removed if they prevented further separation. In addition, the lateral femoral circumflex artery and its branches may prevent further exposure of the lesser trochanter and should be removed if this is the case (Fig. 3C). Intramuscular separation was performed between the iliopsoas and pectineal muscles. Subsequently, the lesser trochanter and tumor were clearly exposed through this channel, and were further exposed by outward rotation of the hip joint. If required, the end of the iliopsoas and pectineal muscles may be removed.

Condition of the patients following surgery. Patients who had received reconstruction of the iliopsoas and pectineal muscle ends had their hip kept in flexor and adductor positions for four to six weeks after surgery. However, patients who did not undergo reconstruction of the muscle ends were able to walk three or four days after surgery.

Statistical analysis. Cadaver measurement data are presented as the mean \pm standard deviation. Statistical analysis was performed using SPSS software, version 19.0 (IBM SPSS, Armonk, NY, USA).

\section{Results}

Surgical outcomes. In the six patients that underwent the novel surgical procedure, the incision lengths ranged between 8 and $12 \mathrm{~cm}$, and the total operative time varied between 60 and $100 \mathrm{~min}$. Continuous epidural anesthesia was used with four patients, while two patients received general anesthesia. The esthetic outcomes were judged to be acceptable, and blood loss ranged between 30 and $200 \mathrm{ml}$ (Table I). The lateral femoral circumflex artery was removed in five patients. In addition, the ends of the iliopsoas and pectineal muscles were resected in one patient and their hip was kept in the flexor and adductor position for five weeks after surgery. The five patients who did not undergo reconstruction of the muscle ends were able to walk three or four days after surgery.

Follow-up and pain. All diagnoses were the same as had been indicated by the biopsies. There were no wound complications and the hip ranges of motion and length were the same as those prior to surgery. No deep vein thrombosis was identified in any of the patients; however, one patient suffered 
Table I. Patient data.

\begin{tabular}{lllccrcc}
\hline Patient & $\begin{array}{c}\text { Age } \\
(\text { years })\end{array}$ & Gender & Anesthesia & $\begin{array}{c}\text { Incision size } \\
(\mathrm{cm})\end{array}$ & $\begin{array}{c}\text { Time } \\
(\mathrm{min})\end{array}$ & $\begin{array}{c}\text { Blood loss } \\
(\mathrm{ml})\end{array}$ & Diagnosis \\
\hline 1 & 21 & Male & CEA & 10 & 75 & 50 & Osteoid osteoma \\
2 & 40 & Female & CEA & 11 & 60 & 30 & Osteochondroama \\
3 & 13 & Female & GA & 8 & 100 & 200 & Osteochondroma \\
4 & 23 & Male & CEA & 12 & 80 & 100 & Osteoid osteoma \\
5 & 25 & Female & CEA & 12 & 60 & 100 & Non-osteogenic fibroma \\
6 & 85 & Male & GA & 12 & 90 & 200 & Liposarcoma \\
\hline
\end{tabular}

CEA, continuous epidural anesthesia; GA, general anesthesia.

Table II. VAS for patient pain prior to and following surgery.

\begin{tabular}{lcc}
\hline & \multicolumn{2}{c}{ VAS (mm) } \\
\cline { 2 - 3 } Patient & Pre-surgery & Post-surgery \\
\hline 1 & 55 & 0 \\
2 & 68 & 0 \\
3 & 57 & 0 \\
4 & 62 & 18 \\
5 & 60 & 0 \\
6 & 43 & 0 \\
\hline
\end{tabular}

VAS, visual analog score.

Table III. Measurements of the space between the femoral artery and femoral nerve.

\begin{tabular}{lccc}
\hline Specimen & Min $(\mathrm{mm})$ & Max $(\mathrm{mm})$ & Mean $\pm \mathrm{SD}(\mathrm{mm})$ \\
\hline $\mathrm{a}$ & 0.00 & 12.72 & $8.01 \pm 2.71$ \\
$\mathrm{~b}$ & 4.86 & 13.48 & $9.65 \pm 2.60$ \\
$\mathrm{c}$ & 5.24 & 14.52 & $10.12 \pm 2.91$ \\
$\mathrm{~d}$ & 4.84 & 13.00 & $9.89 \pm 2.74$ \\
$\mathrm{e}$ & 4.26 & 12.32 & $9.58 \pm 2.86$ \\
$\mathrm{f}$ & 0.00 & 11.50 & $8.39 \pm 2.99$ \\
\hline
\end{tabular}

SD, standard deviation; Max, maximum; Min, minimum.

from sensory dysfunction, with persistent numbness and paresthesia in the distribution of the femur nerve after surgery. These symptoms gradually decreased after three weeks of symptomatic treatment. All six patients were fully satisfied with the extent of pain relief following surgery. Sensations of pain were eliminated entirely in five of the patients following surgery, although a single patient continued to report mild pain (VAS, $18 \mathrm{~mm}$ ) (Table II), while the remaining patient experienced mild pain for six months, which ceased eight to nine months after surgery. No evidence of local tumor recurrence, femur head necrosis or hip joint degeneration disease was identified in any of the patients.
Samples and gap measurement. In total, there were 11 small nerve branches in six of the specimens, three in one specimen and two in three specimens, while the remaining specimens contained just one nerve. Two small arteries and one lymphatic vessel were identified in three separate specimens, respectively. The lateral femoral circumflex artery encounter to femoral nerves in our investigated area in all specimens. The saphenous nerve was the most internal nerve in the selected region; however, in two cases, the nerve was in front of the femoral artery in the proximal region. As two saphenous nerves front the femoral artery in the proximal of our region, in fact, our measurement measured distance from femoral artery trunk lateral margin to medial margin of saphenous nerves in 18 cases, to vastus medialis muscle branches of femoral nerve in two cases. The space in $4 \mathrm{~cm}$ proximal of lesser trochanter was $8.01 \pm 2.71 \mathrm{~mm}, 2 \mathrm{~cm}$ was $9.65 \pm 2.60 \mathrm{~mm}$, $0 \mathrm{~cm}$ was $10.12 \pm 2.91 \mathrm{~mm}$, and $2 \mathrm{~cm}$ distal of lesser trochanter was $9.89 \pm 2.74 \mathrm{~mm}, 4 \mathrm{~cm}$ was $9.58 \pm 2.86 \mathrm{~mm}, 6 \mathrm{~cm}$ was $8.39 \pm 2.99 \mathrm{~mm}$ (Table III). The distance from the highest point of lesser trochanter to the start of the lateral circumflex femoral artery was $12.56 \pm 9.74 \mathrm{~mm}$.

\section{Discussion}

The proximal femur is a common location for benign tumor formation. Current treatment options concentrate on tumors that may lead to hip disfunction, recurring tumors (7) or pediatric cases (4). These procedures typically open the capsule of the hip joint and surgeons select an approach based on the orientation of the tumor (3). Due to the proximity of the lesser trochanter to the capsule of the hip, there is currently no surgical approach aimed specifically at the lesser trochanter. Hip-specific surgical protocols are typically used as the standard approach. Each hip-specific surgical protocol exhibits advantages and disadvantages for performing tumor resection or curettage in the lesser trochanter. A surgical procedure for treating the lesser trochanter more directly may have the advantages of allowing improved exposure to the area, resulting in reduced surgical trauma compared with the conventional hip approach.

The femoral triangle was selected as the operating area due to its location above the lesser trochanter, which offered the shortest distance. The femoral triangle contains a number of vital blood vessels and nerves; thus, conventional surgical 
approaches have typically avoided this region. However, these vital blood vessels and nerves are usually thick, such as the femoral nerve, artery and vein, and can be easily distinguished to avoid injury. When the vital nerves and vessels were crossed, vital nerves and blood vessels were not found in the gap between the trunk of the femoral artery and innermost branch of the femoral nerve. A limited number of small nerve branches and few small artery and lymphatic vessels were observed in the cadaver research. Therefore, following protection of the femoral artery and nerve in the operating region, surgery may be safe and simple.

In the groin, the femoral artery and nerve are separated by the arcus iliopectineus, suggesting that a certain distance should exist in the proximal area of the femoral triangle. Muhly and Orebaugh (8) and Hsu et al (9) used ultrasound to identify a space between the femoral nerve and femoral artery, the size of which is proportional to the rotational angle of the lower limb. The distance is widest at the level of the lesser trochanter (10 samples among 20 specimens) and narrows towards both ends. The spindle region, which has the widest distance in the lesser trochanter, is suitable for the novel surgical procedure. Nerves and vessels are able to be separated appropriately. In addition, enclosed adipose tissue had a cushioning function (10). Thus, it was inferred that an appropriate space could be separated in order to perform surgery, without significant damage to the nerves and blood vessels.

The incision location was easily identified by finding the pulse of the femoral artery. The incision, which followed the outsight of the femoral artery, took advantage of the position of the artery truck to avoid damaging the femoral nerve. The abundance of adipose and muscle tissue was found to protect important nerves and blood vessels, as well as facilitate blunt separation. Adipose tissue between the vessels and nerves was appropriately separated. It was ensured that the femoral nerve and femoral vessels were separated to either side of the channel. Vessels, including arteries and veins, should be trapped and protected towards the medial, while nerves should be pulled laterally. The iliopsoas and pectineal muscles were located deep in the adipose tissue. The lesser trochanter was located at the end of these two muscles, resulting in thinner muscle fiber in this region, which made the lesser trochanter easier to access. In addition, rotation of the hip joint permitted greater exposure. These two muscles are often already separated or pulled to one side by the tumor, typically toward the adipose tissue. The tumors were resected from the adipose tissue. The lateral femoral circumflex artery, which is a main branch of the profunda femoral artery and also a division of the main vessel of the femoral artery, required consideration during surgery. As shown in the cadaveric research, the beginning position of the lateral femoral circumflex artery is varied, and the vessel goes outward so that all the arteries cross the femoral nerve. In the novel surgical approach, the region is further exposed and susceptible to injury. Thus, ligaturing the artery first may be a suitable and safe surgical approach.

The traditional approaches to accessing the lesser trochanter, such as the lateral and anterior, involve extreme rotation of the hip joint. Furthermore, a number of muscles are typically removed, and reconstruction is required. These factors contribute to increased surgical trauma and an increased frequency of complications following surgery. The medial approach is closely compared with other traditional approaches; however, the muscles and vessels in that region are even more complex (11). A number of nerves and blood vessels are present around the adductor brevis, including the obturator nerve, artery and vein, which innervate and supply muscles, such as the adductor longus, adductor magnus and gracilis. Moreover, high anatomical variations in these nerves, blood vessels and their branches increase the risk of injury (6). Medial approaches are often used to treat dysplasia or hip dislocation; however, this approach has been shown to have an avascular necrosis rate of 12-54.3\% (12-16). Furthermore, the incision is close to the perineum, which increases the risk of infection.

Due to the decreased distance between the point of incision and destination and careful separation in the novel surgical approach, the surgery time, trauma and blood loss were minimal. In addition, the complications caused by long periods of immobility following surgery may be limited since patients can exercise earlier following the procedure. The follow-up assessment revealed evident pain relief and no evidence of lesion recurrence, indicating that the surgical resections performed in the present study were successful. The results of the VAS revealed that the majority of patients experienced complete pain relief, with only one patient continuing to report mild pain. However, the cause of this remaining pain is not clear. We hypothesize that the nerve or vessel was trapped for an extended period of time. Full exposure of the tumor site is a basic condition for resective surgery. Due to the absence of lesion recurrence, the exposed area was considered to be sufficient. The separation process was relatively simple, which was reflected by the short surgical time and decreased blood loss. The extent of blood loss was associated with the lesion size and the degree of adhesion of the surrounding tissue. A longer surgical duration may lead to greater blood loss.

Two potential complications were identified prior to surgery. Firstly, femur head necrosis and hip joint degeneration disease may occur as a result of removing the lateral femoral circumflex artery or its branches ( $5 / 6$ patients), since it is one of the main supply arteries of the femur head. However, no such problem was identified during follow-up. It was inferred that the blood supply was compensated by the numerous anastomotic vessels near the greater trochanter, whose branches supply $65-80 \%$ of blood to the femoral head (17-21). However, the long-term risk of femoral head degeneration is not known. The other possible complication may result from the blood vessels and nerves having been separated for an extended period of time. Separating blood vessels increases the risk of thrombus development; however, no evidence of a blood vessel thrombus was identified following surgery, which may be a result of the limited sample size. The femoral nerve was also kept tense during surgery, and a number of its small branches may be removed. This may cause sensory dysfunction, with persistent numbness and paresthesia. Only one patient suffered from these symptoms, which decreased three weeks after surgery. Irreversible nerve damage requires further study, as the sample size was small in the present study. Although small branches of the femoral nerve were removed, knee strength was not decreased. Thus, it was hypothesized that the flexor muscle group may not be impaired. 
In conclusion, the novel approach described in the present study may be a feasible protocol for the resection of benign tumors, and also the preoperative palliative resection of malignant tumors. The technique is particularly suited to tumors extending toward the lateral and anterior of the lesser trochanter.

\section{References}

1. Barros Filho TE, Oliveira RP, Taricco MA and Gonzalez CH: Hereditary multiple exostoses and cervical ventral protuberance causing dysphagia. A case report. Spine (Phila Pa 1976) 20 1640-1642, 1995.

2. Cardelia JM, Dormans JP, Drummond DS, Davidson RS Duhaime C and Sutton L: Proximal fibular osteochondroma with associated peroneal nerve palsy: A review of six cases. J Pediatr Orthop 15: 574-577, 1995

3. Bottner F, Rodi R, Kordish I, Winklemann W, Gosheger G and Lindner N: Surgical treatment of symptomatic osteochondroma. A three- to eight-year follow-up study. J Bone Joint Surg Br 85: 1161-1165, 2003.

4. Learmonth DJ and Raymakers R: Osteochondroma of the femoral neck secondary to a slipped upper femoral epiphysis. Arch Orthop Trauma Surg 112: 106-107, 1993.

5. Li M, Luettringhaus T, Walker KR and Cole PA: Operative treatment of femoral neck osteochondroma through a digastric approach in a pediatric patient: a case report and review of the literature. J Pediatr Orthop B 21: 230-234, 2012.

6. Anagnostopoulou S, Kostopanagiotou G, Paraskeuopoulos T, Chantzi C, Lolis E and Saranteas T: Anatomic variations of the obturator nerve in the inguinal region: Implications in conventional and ultrasound regional anesthesia techniques. Reg Anesth Pain Med 34: 33-39, 2009.

7. Hsieh PC, Ondra SL, Grande AW, et al: Posterior vertebral column subtraction osteotomy: a novel surgical approach for the treatment of multiple recurrences of tethered cord syndrome. J Neurosurg Spine 10: 278-286, 2009.

8. Muhly WT and Orebaugh SL: Ultrasound evaluation of the anatomy of the vessels in relation to the femoral nerve at the femoral crease. Surg Radiol Anat 33: 491-494, 2011.
9. Hsu HT, Lu IC, Chang YL, Wang FY, Kuo YW, Chiu SL and Chu KS: Lateral rotation of the lower extremity increases the distance between the femoral nerve and femoral artery: An ultrasonographic study. Kaohsiung J Med Sci 23: 618-623, 2007.

10. Norgan NG: The beneficial effects of body fat and adipose tissue in humans. Int J Obes Relat Metab Disord 21: 738-746, 1997.

11. Moore AE and Stringer MD: Iatrogenic femoral nerve injury: A systematic review. Surg Radiol Anat 33: 649-658, 2011.

12. Szöke G, Staheli LT, Jaffe K and Hall JG: Medial-approach open reduction of hip dislocation in amyoplasia-type arthrogryposis. J Pediatr Orthop 16: 127-130, 1996.

13. Tumer Y, Ward WT and Grudziak J: Medial open reduction in the treatment of developmental dislocation of the hip. J Pediatr Orthop 17: 176-180, 1997

14. Tarassoli P, Gargan MF, Atherton WG and Thomas SR: The medial approach for the treatment of children with developmental dysplasia of the hip. Bone Joint J 96-B: 406-413, 2014.

15. Koizumi W, Moriya H, Tsuchiya K, Takeuchi T, Kamegaya M and Akita T: Ludloff's medial approach for open reduction of congenital dislocation of the hip. A 20-year follow-up. J Bone Joint Surg Br 78: 924-929, 1996.

16. Okano K, Yamada K, Takahashi K, Enomoto H, Osaki M and Shindo H: Long-term outcome of Ludloff's medial approach for open reduction of developmental dislocation of the hip in relation to the age at operation. Int Orthop 33: 1391-1396, 2009.

17. Kalhor M, Horowitz K, Gharehdaghi J, Beck M and Ganz R: Anatomic variations in femoral head circulation. Hip Int 22 307-312, 2012.

18. Ichikawa T, Haradome $\mathrm{H}$, Hachiya J, Nitatori $\mathrm{T}$ and Araki T: Diffusion-weighted MR imaging with a single-shot echoplanar sequence: detection and characterization of focal hepatic lesions. AJR Am J Roentgenol 170: 397-402, 1998.

19. Grose AW, Gardner MJ, Sussmann PS, Helfet DL and Lorich DG: The surgical anatomy of the blood supply to the femoral head: description of the anastomosis between the medial femoral circumflex and inferior gluteal arteries at the hip. J Bone Joint Surg Br 90: 1298-1303, 2008.

20. Boraiah S, Dyke JP, Hettrich C, Parker RJ, Miller A, Helfet D and Lorich D: Assessment of vascularity of the femoral head using gadolinium (Gd-DTPA)-enhanced magnetic resonance imaging: a cadaver study. J Bone Joint Surg Br 91: 131-137, 2009.

21. Gautier E, Ganz K, Krügel N, Gill T and Ganz R: Anatomy of the medial femoral circumflex artery and its surgical implications. J Bone Joint Surg Br 82: 679-683, 2000. 